Cours de formation post-graduée et continue de la SSDV

Méthodes de traitements physiques en dermatologie, Lausanne, 13 et 14 mai 2004

\title{
Bulletin d'inscription
}

(s.v.p. en caractères d'imprimerie ou tampon)

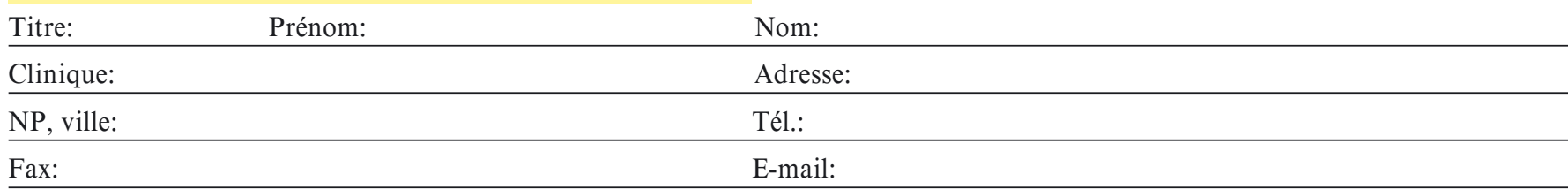

Année de formation:

Activité: $\square$ Clinique $\square$ Cabinet $\square$ Autre

Je m'inscris au cours sus-mentionné: jeudi, 13 mai $\square$ vendredi, 14 mai $\square$

Frais du cours: $\quad$ médecins hospitaliers (assistants)

CHF 300,médecins installés CHF 400,-

$\square \mathrm{CHF}$ carte journalière CHF 200,-

$\square \mathrm{CHF}$ carte demi-journée

CHF 100,-

CHF 20,-

$\square \mathrm{CHF}$

CHF 20,-

$\square \mathrm{CHF}$

$\square \mathrm{CHF}$

Je désire prendre part au lunch du 13 mai

Je désire prendre part au lunch du 14 mai

Montant total à verser sur le CCP 10-1900-8,

$\square \mathrm{CHF}$

$\mathrm{CHF}$

Hospices cantonaux/CHUV, 1011 Lausanne, avec mention CGRB 26644, congrès SSDV

Merci d'envoyer ou faxer ce bulletin d'inscription pour le 6 mai 2004 au plus tard à

Secrétariat, Service de dermatologie, Hôpital de Beaumont, Beaumont 04, CH-1011 Lausanne/CHUV, Fax 0213140382

Weiter- und Fortbildungskurs der SGDV

Physikalische Behandlungsmethoden in der Dermatologie, Lausanne, 13. und 14. Mai 2004

\section{Anmeldeformular}

(Bitte gut leserlich mit Blockschrift ausfüllen bzw. Stempel)

\begin{tabular}{|c|c|}
\hline Vorname: & Name: \\
\hline Klinik: & Adresse: \\
\hline Ort mit Postleitzahl: & Tel.: \\
\hline Fax: & E-Mail: \\
\hline
\end{tabular}

Weiterbildungsjahr:

Tätigkeit: $\square$ Klinik $\square$ Praxis $\square$ Sonstiges

Hiermit melde ich mich für den Kurs an: Donnerstag, 13. Mai $\square \quad$ Freitag, 14. Mai $\square$

$\begin{array}{llll}\text { Kurskosten: } & \text { Assistenten } & \text { CHF 300.- } & \square \text { CHF. } \\ & \text { Niedergelassene Dermatologen } & \text { CHF 400.- } & \square \text { CHF } \\ & \text { Tageskarte } & \text { CHF 200.- } & \square \text { CHF } \\ \text { 1/2-Tageskarte } & \text { CHF 100.- } & \square \text { CHF } \\ & \text { CHF 20.- } & \square \text { CHF } \\ \text { Ich möchte teilnehmen am Lunch vom 13. Mai } & \text { CHF 20.- } & \square \text { CHF } \\ \text { Ich möchte teilnehmen am Lunch vom 14. Mai } & & \text { CHF }\end{array}$

Hospices cantonaux/CHUV, 1011 Lausanne, CGRB 26644, Congrès SSDV

Anmeldeformular bis spätestens 6. Mai 2004 einsenden an:

Secrétariat, Service de dermatologie, Hôpital de Beaumont, Beaumont 04, CH-1011 Lausanne/CHUV, Fax 0213140382 